\title{
Monitoring disease in England and Wales: methods applicable to routine data-collecting systems
}

\author{
PATRICIA FRASER, VALERIE BERAL, AND CLAIR CHILVERS \\ From the Epidemiological Monitoring Unit, Department of Medical Statistics and Epidemiology, London \\ School of Hygiene and Tropical Medicine
}

SUMMARY This paper reviews routine data-collecting systems and methods for disease surveillance in England and Wales. It discusses population-based correlation studies, which seek to explain disease trends by relating routine health statistics to possible causative agents on a secular, geographical, or occupational basis. It describes recent developments in linking information collected by routine general purpose systems as a means of identifying and following individuals exposed to potential hazards.

The establishment of the Epidemiological Monitoring Unit was the last major research venture undertaken by the late Professor D. D. Reid. The unit was set up in October, 1976, six months before his death. He had always believed that routinely collected health statistics were a relatively unexploited source of information in epidemiological research. True to his origins, he was attracted by the economy effected in using existing data to suggest and test hypotheses about disease causation. He envisaged that the Epidemiological Monitoring Unit would have three main functions-to review current sources of routinely collected data and the uses made of them in monitoring disease; to develop methods of analysis which would result in the early recognition of new health hazards; and to relate health statistics to routine sources of information on possible causative agents for testing specific hypotheses. This paper attempts to meet the first of these objectives.

Monitoring aims to detect and explain any unexpected deviation in disease trends or distribution. The detection or surveillance of trends originated in the seventeenth century, when Bills of Mortality were first used to draw attention to the presence of certain diseases in London. The term 'surveillance' was originally restricted to the follow-up of cases and contacts of infectious disease, but it now refers to observation of disease as well as of individuals. Langmuir (1963) defined surveillance as 'the continued watchfulness over the distribution and trends of incidence through the systematic collection, consolidation and evaluation of morbidity and mortality reports and other relevant data.' More recently the term has also been applied to evaluating the performance of health services (Doll, 1974).

Monitoring does not end with a description of disease trends or distribution: it also seeks an explanation. In monitoring infectious disease, identifying the pathogen or positive serology confirms exposure and may establish the link between the causative organism and its effect. In non-communicable disease it is often more difficult to establish a link between cause and effect, because of long latent intervals and the operation of multiple causes.

\section{Data collection}

The first step in any monitoring system is the systematic collection of data. England and Wales are well served by the quality and content of information on disease that is routinely available, but these data tend to be dispersed in separate reports by the various government departments concerned. The records of the General Register Office (GRO), reconstituted as the Office of Population Censuses and Surveys (OPCS) in 1970, are the most valuable source of information on population, mortality, and morbidity (Adelstein, 1976). The Department of Health and Social Security (DHSS) holds records of sickness absence, admissions to mental hospitals, and registers of handicapped persons. The Department of Education and Science (DES) reports on the health of schoolchildren.

Estimates of population size, essential to provide denominators for calculation of rates, are derived from the census. Death certification and the notification of certain infectious diseases and abortion 
are governed by statutory instruments, but the remaining systems for the collection of routine health statistics in England and Wales rely on voluntary co-operation. The main features of these systems are summarised here. A comprehensive review is provided by Alderson and Whitehead (1974).

\section{DEATH CERTIFICATION}

Mortality records, based on mandatory registration of deaths and stillbirths, include medical certification of the causes of death. A number of studies have examined the accuracy of death certification, usually by comparison with data derived from necropsy or with detailed case histories. Agreement varies with the cause of death. Alderson (1965) compared the information available from a detailed case history with the wording on the death certificate and found that for $90 \%$ of the deaths there was no major discrepancy. Three different studies have indicated that the death rate from all cancers is under-certified by about $5 \%$ (Wynne-Griffith, 1976). Some efforts are now being made to avoid statistical artefacts in mortality data by coding multiple causes, so that use can be made of information on the death certificate other than the underlying cause.

\section{CANCER REGISTRATION}

Cancer registration began in England and Wales in 1939 but it was not until 1962 that all the hospital regions had joined the national scheme. Now data are forwarded to the OPCS under voluntary arrangements from regional centres registering cancer cases notified to them by hospitals. Although there is evidence that the proportion of total cases registered is increasing, comprehensive coverage has not yet been achieved. A recent inquiry showed that some registries are able to provide detailed information on the occurrence of cancer in their region, while others are operating with the minimum of staff and equipment. This means that data is not available for research, and delay in forwarding registrations to the OPCS also delays the publication of national statistics. In 1978, the most recent published data relate to 1970 .

\section{HOSPITAL INPATIENT STATISTICS}

Compilation of hospital inpatient statistics began in 1929, and the introduction of the National Health Service (NHS) in 1948 provided the opportunity for a national scheme for collecting hospital morbidity data. After unsuccessful attempts to obtain complete coverage, the inquiry was restricted to a one in ten sample of hospital deaths and discharges. It was proposed at first to include names and NHS numbers so that records of successive admissions could be linked, but this was not implemented nationally on the grounds that it would breach the confidentiality of medical records. A system linking records of birth, death, hospitalisation, and obstetric delivery has been operating in the Oxford area since 1963 (Acheson, 1967). An estimate of the number of patients suffering from mental disorders in all NHS psychiatric hospitals and units in England and Wales is provided by the Mental Health Enquiry.

\section{NOTIFICATION OF INFECTIOUS DISEASES}

Compulsory notification of certain infectious diseases began in England and Wales in 1876, and by 1899 notification had become obligatory throughout the country. However, the completeness of notification is variable; it is very poor for some of the milder infections. Although incomplete, notification of cases of infectious disease continues to be a useful source of information for detecting sudden changes in disease occurrence.

\section{NOTIFICATION OF ABORTION}

The Abortion Act was introduced in 1968. This required notification of termination of pregnancy to be made within seven days to the DHSS. There is no system advocated for checking these certificates at local or regional level.

\section{NOTIFICATION OF CONGENITAL MALFORMATIONS}

In 1961 the teratogenic action of thalidomide became apparent and the drug was promptly withdrawn from the market. This tragedy was the stimulus for the development of a national scheme for the voluntary notification of congenital malformations in newborn babies in England and Wales. Notification is fairly complete but there is some variation from one local authority to another.

\section{NOTIFICATION OF ADVERSE REACTIONS TO DRUGS}

The Committee on Safety of Medicines keeps a register of suspected adverse drug reactions. It asks medical practitioners to complete a 'Yellow Card' reporting all reactions to new drugs, and serious or unusual reactions to established drugs, including vaccines. On the basis of this information, the committee publishes a warning in the Adverse Reactions Series, or under Current Problems if it is intended to draw attention to problems requiring further investigation before a definitive statement is made.

\section{OTHER SOURCES OF MORBIDITY DATA}

Two national surveys in general practice, and data forwarded to the General Practice Research Unit, 
provide information on consultations for disease and disability in the general population. Sickness absence certificates give some indication of the minor ailments suffered by the working population, and school medical examinations provide information on the health of schoolchildren. The General Household Survey requests information on sickness causing limitation of activity, and on visits to doctors and hospitals. Local authorities are expected to gather information about the scale and needs of chronic sick and disabled persons in their area. The data are collected centrally but the registers of blind and partially-sighted persons, of deaf persons, and of general handicap, tend to be very incomplete.

Sources of morbidity data collected on a regular basis provide some information about the level and type of disease and disability in the community. Their coverage is less complete and the data generally less accurate than mortality statistics. Interpreted with due regard to their limitations, they supplement mortality statistics for fatal diseases, and necessarily supplant them in the surveillance of non-fatal conditions.

\section{Disease surveillance}

\section{TIME TRENDS}

The analysis of time trends is fundamental in disease surveillance. Differences in death rates over time may be apparent, but the crucial question is whether they reflect real changes in disease occurrence. Death rates may be affected by alterations of disease definition and classification, of case-finding methods and survival, and of fashion or convention in the completion and coding of death certificates (Reid and Grimley Evans, 1970). In spite of the problems of interpreting time trends in death rates, their indications are often valid, yet unwisely ignored. Neglect of available information led, for example, to an accumulation of over 73000 deaths from lung cancer in England and Wales between 1916 and 1950 (Royal College of Physicians, 1962). Similarly, although there were vital statistical reports of lethal episodes of air pollution at intervals throughout the preceding 100 years, it was not until the 1952 episode, when over 5000 people died in London, that comprehensive studies were undertaken (Ministry of Health, 1954). Retrospective inspection of vital statistical data on these topics emphasised that trends in mortality from these diseases were evident long before specific research was undertaken.

\section{LONG-TERM TRENDS}

The study of long-term trends in disease is a basic aspect of epidemiological analysis. For this purpose, mortality rates, recorded in England and Wales since 1841, provide the most detailed and useful source of data and have recently been reviewed (OPCS, 1978a). Marked changes in disease frequency have been observed during this century, with mortality rates from circulatory disease and neoplasms increasing substantially, and mortality rates from infectious diseases and maternal causes showing a dramatic decline.

Since 1841, the Registrar General's annual reports have published mortality data subdivided by cause, age, and sex. For recent years, the OPCS publication 'Mortality Surveillance, England and Wales, 19681976' (OPCS, 1978b) lists annual deaths and death rates by sex and age for the 150 causes of death in the abbreviated A-list of the International Classification of Diseases (ICD) (World Health Organisation, 1967). Statistical tests are performed to indicate significant changes in the annual death rates for each cause of death during the nine-year period. This surveillance system focuses attention on a subset of diseases showing recent changes in mortality, in some or all age groups, which may warrant further investigation.

An increasing interest in the study of serial data on cancer mortality during the last decade led to the publication of 'Cancer Mortality, England and Wales, 1911-1970' (OPCS, 1975). This publication presents the number of deaths and death rates for five-year age groups by sex, site, and five-year calendar periods. The Institute of Cancer Research (1976) has published more complete serial mortality tables covering the same period for the whole of the British Isles. Cancer registration data from a large area in the Midlands, where cancer registration has been nearly complete for more than 12 years, is the source of a recent publication on trends in incidence and survival (Waterhouse, 1974). Comparable data are not available for England and Wales as a whole.

While age- and sex-specific rates provide the most valuable and detailed information, it is often desirable to have a single summary measure to describe the whole experience of a population, adjusting for differences in the distribution of factors known to influence mortality and morbidity. Two commonly used methods, direct and indirect standardisation, are both greatly influenced by the number of deaths in the older age groups. Other measures of mortality have been proposed which place greater emphasis on mortality in younger age groups. Yerushalmy (1951) constructed a mortality index on the principle that equal proportionate changes should affect the index equally, no matter at which point in the age scale the changes occur. Kleinman (1977) has criticised this approach on the 
grounds that it gives too much weight to small numbers of deaths which may be unstable.

To rely on conventional death rates as a measure of disease in the community would lead to the conclusion that our most pressing health problem is chronic disease in old age. This may be so, but it should not be allowed to overshadow the importance of the years of active life lost by death at younger ages. Alternative methods for measuring mortality which take into account the potential years of life lost have been suggested (Haenszel, 1950). The introduction of an allowance for years of life lost alters the relative ranking of important causes of death by increasing markedly the influence of causes such as accidents and congenital anomalies which are responsible for many deaths at younger ages. These measures are worthy of further study in the context of disease surveillance and allocation of priorities in research and health care.

\section{SEASONAL TRENDS}

In England and Wales the overall death rate fluctuates markedly by season, with higher rates in winter than in summer, due to periodic fluctuations in many conditions, both infectious and non-infectious. There is, for example, a marked seasonal distribution of acute respiratory viral infections, with an increase in deaths attributed to respiratory causes in winter months. An association between cold winter weather and short-term increases in mortality from ischaemic heart disease has also been noted (Rose, 1966).

Special problems of surveillance arise in a condition such as influenza, which is not notifiable, and where mortality attributed to the disease underestimates its effect on the population. The concept of 'excess mortality' associated with influenza outbreaks has been used to provide a more realistic figure. Recently Clifford et al. (1977) have examined the relationship between notified mortality, new sickness benefit claims, and a number of variables thought likely to influence these notifications. They obtained reasonable estimates of both mortality and morbidity from influenza in the working population, and concluded that the 'excess mortality' associated with influenza was considerable, even in the absence of major epidemics.

Since 1964, a surveillance system has been operating in England and Wales for the early detection of any change in the frequency of reporting of congenital malformations (Weatherall and Haskey, 1976). Some abnormalities show a seasonal variation in incidence. For example, more cases of anencephalus are notified during winter months (Leck and Record, 1966). The OPCS routinely surveys the notifications of some 60 groups of malformations from 98 area health authorities in England and Wales. Monthly tabulations give the numbers of each type of malformation and the numbers of babies with combined defects. These tabulations are sent as a return to area medical officers. Two statistical tests are used to help distinguish true changes, due to external factors, from chance fluctuations. Although designed primarily for the early detection of congenital malformations, this system also provides the basis for studying the occurrence of malformations in relation to environmental factors suspected of being teratogenic or of causing other toxic effects. Notifications are also used to monitor the effect of changes in therapeutics or in case-finding, and individual cases may provide a starting point for family studies to assess the role of genetic influences (Weatherall, 1978).

\section{WEEKLY TRENDS}

Communicable disease surveillance relies heavily on the prompt dissemination of information. In England and Wales, surveillance over notifiable infections is maintained routinely by the OPCS. Notifications of infectious disease are published weekly by area, and statistical tests for significant excesses give a clear picture of the geographical distribution of epidemics.

The Communicable Disease Surveillance Centre was set up in 1977. One of its functions is to provide up-to-date information quickly on the current state of communicable disease in England and Wales (Galbraith and Berrie, 1978). It does so by publishing the weekly Communicable Disease Report, compiled from information received in confidential reports from public health and hospital laboratories.

The weekly returns submitted to the General Practice Research Unit of the Royal College of General Practitioners are an additional source of routine information on communicable and respiratory diseases. The returns cover a population of about 200000 in 40 general practices and report newly diagnosed episodes of communicable and respiratory diseases.

\section{DAILY TRENDS}

Farr (1868) used daily totals of deaths in London during cholera epidemics to locate an infected water supply, but daily death totals were not analysed again in England until after the London fog in 1952 (Ministry of Health, 1954). Since 1969, daily death totals from all causes, and from cardiovascular, cerebrovascular, and respiratory diseases, have been tabulated for conurbations in England and Wales. These daily totals are particularly useful 
when less frequent reporting is too insensitive to detect sudden short-term changes in mortality. Macfarlane (1977) has used daily death totals to examine the association between mortality, air pollution, and temperature in Greater London. In this type of study, time series analysis is often needed to separate daily fluctuations in mortality from seasonal and long-term trends.

\section{GEOGRAPHICAL ANALYSIS}

At regular intervals since 1848 the Registrar General has reviewed area mortality in England and Wales. Mortality statistics for standard regions, conurbations, urban and rural aggregates, hospital regions, and other subdivisions of the standard regions, are presented in the annual review and supplemented by a full decennial analysis.

The standardised mortality ratio (SMR) has been used extensively for the presentation of area statistics. Although SMRs permit valid comparisons of mortality between regions, their value for temporal comparisons is limited by change of age structure within regions over time, administrative changes in boundaries, and revision of the ICD (International Classification of Diseases). In order to make mortality data more readily available to research workers, the 1971 decennial supplement will include tabulations on microfiche for various regional subdivisions and selected causes of death. A commentary will be published separately. Meanwhile, the mortality differences between the regions of England and Wales in 1969-73 have been summarised for broad groups of causes of death (Chilvers, 1978) and for certain malignant neoplasms (Chilvers and Adelstein, 1978).

In general, mortality experience, heavily influenced by deaths from circulatory disease, is less favourable in the northern regions of England and in South Wales than in the south of England. Mortality is low in rural areas and increases steadily with increasing urbanisation. The regional distribution of mortality is similar to that of adults in both infants and children, although congenital anomalies and accidents are the major causes of death in these age groups.

In addition to the decennial supplements and the regional statistics in the Registrar General's annual reviews, the OPCS also produces unpublished annual tabulations of the number of deaths by age, sex, and local administrative area for certain causes of death. From 1975, these statistics will be cumulated for an extended list of causes of death, providing a valuable source of mortality data for these areas.

There are particular difficulties in interpreting mortality data from small areas. The numbers of deaths from any cause fluctuate; and even when deaths over five years are aggregated, the number in an area may be so small that a few additional deaths may result in spuriously high rates. Yet, if area mortality data are to be useful in identifying localised environmental and other health hazards, it is necessary to consider small areas. In the United States, mortality statistics have been analysed extensively by county and state economic area to provide clues to disease causation (Hoover et al., 1975). A similar approach might usefully be employed in England and Wales.

\section{OCCUPATIONAL ANALYSIS}

Since 1861, occupational mortality has formed an integral part of the series of supplements which review mortality every ten years in greater depth than is possible in the Registrar General's annual reviews. The earliest decennial supplements concentrated on direct occupational influences on mortality. Later, attention turned to differences between social classes, defined on the basis of occupation, which reflected varying social environments and different life styles. This series of supplements provides the only regular authoritative analysis of mortality in relation to occupation and social class. The review serves as a standard reference for investigators who suspect an association between an occupation and mortality from a particular cause of death, and provides valuable clues for further study.

The 1971 review, covering deaths in the three years 1970-72, is the twelfth in the series (OPCS, 1978c). The main findings have been summarised (Fox, 1977). Initially the review concentrates on broad social groups derived from the standard classification of occupations by social class. For the first time, mortality at all ages under 75 for both sexes is covered, providing information on social class differences in infant, childhood, and adult mortality. Differences between classes are clear and regular at all ages; Social Class V usually has the highest mortality rates, and these decline regularly to Social Class I. An alternative grouping of occupations into socioeconomic groups gives an indication of variations in mortality within classes.

The Health and Safety Executive (HSE) (1976) specifies limits for chemical substances, mineral dust, and nuisance particulates in workroom air which should not be exceeded. The HSE maintains surveillance over workers exposed to certain recognised hazards, such as lead and asbestos.

\section{Measuring exposure}

The importance of environmental factors in disease causation is now widely recognised. Individuals 
may be exposed to known or potential hazards through their personal habits and way of life, in the environment, or at work. Population-based correlation studies, aiming to relate mortality and morbidity to the level of exposure to suspected risk factors, use estimates of the overall level of exposure of the population rather than precise measures of individual exposure. Correlation studies are feasible only when reliable statistics on exposure have been compiled. The Guide to Official Statistics (1978) offers detailed information on all official and some important non-official sources published during the last decade. Official sources include the National Diet Survey and the Annual Abstract of Statistics. Non-official sources include the Tobacco Research Council, which, until its recent dissolution, provided comprehensive information on smoking, and Intercontinental Medical Statistics Ltd., which estimates total sales of drugs.

In England and Wales there are several regular sources of regional statistics, additional occasional reports, and regional breakdowns in other publications such as the General Household Survey. They provide information on regional differences in diet, alchohol, and cigarette consumption and other aspects of people's ways of life. Unfortunately, health statistics for small areas can seldom be related to exposure data covering comparable areas.

Many organisations are measuring substances in the environment to assess present levels and predict future trends. The National Survey on Air Pollution, for example, has been monitoring air pollutants for the last 20 years. In 1965 the Natural Environment Research Council was established, and it supports research on monitoring air, marine, and freshwater pollution. Since 1974, the Water Research Centre has been particularly concerned with water quality in relation to health. The Department of the Environment, the Ministry of Agriculture, Fisheries and Food, and the Monitoring and Assessment Research Centre are all actively involved in measuring environmental pollutants.

\section{Testing hypotheses}

\section{CORRELATION STUDIES}

Study of secular trends in disease in relation to changes in suspected risk factors has suggested many links between cause and effect. For example, the rising tide of drunkenness offences, and of hospital admissions and deaths due to alcoholism and cirrhosis, parallels the increase in alcohol consumption since the second world war, and emphasises the increase in alcoholism in England and Wales (Donnan and Haskey, 1977). A study of the increase in hospital admissions and consultations in general practice for ectopic pregnancy during the late 1960 s and early 1970 s indicated that the increasing use of intrauterine contraceptive devices was probably responsible (Beral, 1975).

When exposure to a factor in early life is thought to be important in disease causation, comparison of mortality rates in successive birth cohorts may reveal important mortality patterns, which are often obscured by straightforward analysis of time trends. For example, against a background of declining mortality from cervical cancer in England and Wales, the generations who were young adults during the second world war were noted to be experiencing higher mortality from this cancer than were other generations of women (Hill and Adelstein, 1967). Beral (1974) went on to test the hypothesis that cervical cancer is related to a venereally-transmitted agent by examining standardised cohort mortality ratios from cervical cancer in relation to the incidence of gonorrhoea. The stability of the trends observed strongly suggested that cervical cancer follows, at a variable interval, exposure to genital infection in early life. More recently, cohort analysis has indicated a clear inverse relationship between average completed family size and mortality from ovarian cancer in different populations of women (Beral et al., 1978).

Comparison of disease experience in different countries is useful in confirming the consistency of trends and in investigating the cause of disease. For example, such studies demonstrated the association between mortality from arteriosclerotic heart disease and from lung cancer in relation to national levels of cigarette consumption (Reid, 1975). More recently, analysis of international trends in cardiovascular mortality among young women has revealed a close association with changes in the prevalence of oral contraceptive use in each country (Beral, 1976).

Geographical correlation studies in England and Wales have linked patterns of mortality and morbidity with suspected risk factors. For example, studies of county boroughs in England and Wales have suggested a strong association between cardiovascular mortality and soft drinking water (Crawford et al., 1971; Elwood et al., 1977). In another comparison between county boroughs, hospital discharge rates were used to demonstrate the adverse effect of high air pollution on the incidence of bronchitis and middle ear disease (Reid, 1969). In a study of 10 towns in northern England, it was suggested that high nitrate concentration in the drinking water in one of them was related to an increased risk of death from gastric cancer (Hill et al., 1973).

Studies of even smaller geographical areas may reveal considerable variation in the incidence of 
disease, which may be related to environmental factors. For example, in a study of the cause of death by parish in west Devon over a period of 20 years, an uneven distribution of cancer deaths was noted (Allen-Price, 1960). This was attributed to the diverse water supplies, and the author put forward the hypothesis that the causative agent was water-borne. More recently, respiratory cancer clustering has been reported in association with localised air pollution (Lloyd, 1978).

Investigation of common characteristics shared by occupations with similar mortality patterns may elucidate the influence of exercise, indoor versus outdoor labour, and damage caused by dust, dirt, polluted air, and noise. Occupational mortality, when related to other differences between occcupations, may help to explain disease causation. For example, smoking data from the General Household Survey have been linked to mortality recorded for occupational groups in the decennial supplements (OPCS, 1978c). This study demonstrated that occupations with a high proportion of smokers tended to have high mortality from lung cancer.

\section{MIGRANT STUDIES}

Studies of migrants afford an opportunity to investigate the impact on health that changes in living environment might bring. Study of mortality among migrant populations has been particularly rewarding in the fields of cancer research and cardiovascular disease (Reid, 1975). In England and Wales, information on birthplace has been included on the death certificate since 1969. This innovation enables the OPCS to study mortality according to birthplace of the deceased, or, for infants, the birthplace of parents (Adelstein, 1976).

\section{RECORD LINKAGE STUDIES}

Recently the OPCS has been linking information collected by routine general-purpose data-collecting systems (Adelstein, 1976). A study has been undertaken in which, for a one per cent sample of the population, data held by the OPCS about separate events occurring to an individual are being linked by use of the National Health Service Central Register (NHSCR) (OPCS, 1973). The system is designed to reveal relationships of characteristics noted in the census, and at birth registration, with subsequent events such as cancer registration and causes of death. Although these are events about which information is already gathered by the OPCS, additional steps are being taken to safeguard confidentiality.

A system of linked medical records makes possible the study of relationships between successive events in a patient's medical history. For example, in the Oxford area, such a system has been used to compare the principal diagnoses on discharge from hospital in successive periods of inpatient care (Hobbs et al., 1976). Disease associations were demonstrated, and this study provided the opportunity for a systematic appraisal of the hospital precursors of malignant disease.

Samples of linked records can be chosen to test a specific hypothesis. For example, census schedules can be used to identify individuals in certain occupations who may be exposed to potentially noxious agents. Records of such persons can be traced in the NHSCR where all deaths and cancer registrations are recorded routinely. Follow-up information can thus be obtained as part of the routine work involved in keeping the register up to date. These studies are carried out by the OPCS without divulging the subjects' identities to investigators. They offer a powerful tool for epidemiological inquiry because, by linking exposure to outcome on an individual basis, they may provide direct evidence of causation. This type of prospective study is relatively inexpensive, and simpler to perform than more traditional studies, which generally require the interviewing, examination, and follow-up of large numbers of individuals over long periods of time.

\section{Conclusion}

To maintain surveillance over disease in the community, recourse has to be made to routine data-collecting systems. In general, mortality statistics are sufficiently reliable, but morbidity statistics must be interpreted with due regard to their limitations if sensible conclusions are to be drawn. There is a need for a continuing review of all relevant data, for the consistency of trends in data from different sources often provides strong evidence that apparent changes in disease frequency are real. Many 'epidemics' have been detected through a clinician's awareness of the changing pattern of a disease, rather than through routine surveillance systems. Increases in rare diseases are particularly likely to be missed by routine systems, because they tend to be lost in the broad categories used to classify disease, region, or occupation.

The number and amount of chemicals manufactured and used is increasing greatly, so it must be assumed that new hazards will continue to be introduced. Their acute toxic effects may be obvious, but when they are absorbed in small doses over long periods of time their adverse effects may well pass unnoticed. There are many instances where exposure to certain hazards at work is likely to be much greater than it is elsewhere. Studies of mortality amongoccupational groups exposed to comparatively 
high levels of toxic substances may indicate the possible implications for the general population exposed to lower levels. New developments in linking records from routine sources offer a relatively inexpensive method for relating exposure to outcome in occupational groups.

Potentially harmful substances released into the environment, such as air pollutants or contaminants of drinking water, generally show an uneven geographical distribution. It is thus possible to examine their effect by comparing disease patterns in populations exposed to different levels. The scope for geographical correlation studies is limited by the availability of comparable data on disease and exposure. There is a need for all geographical statistics to be compiled on a common basis to enable valid comparisons to be made.

In seeking for causes of disease, the evidence provided by population-based correlation studies is circumstantial. While an association does not provide direct evidence of causation, it nevertheless indicates possible risk factors and communities worthy of further study.

Definite evidence of cause and effect relationships can only be provided by individual-based studies. Since the latent period between exposure to a hazard and the onset of disease may be long, one of the limitations of prospective studies has been the difficulty and expense of following individuals over long periods. Recent developments in linking records from routinely available sources should help to overcome this difficulty. At present, the bar to their full exploitation lies in the limitation of resources and the need to protect privacy.

This work is supported by a grant from the Medical Research Council.

Reprints from Dr. Patricia Fraser, Epidemiological Monitoring Unit, Department of Medical Statistics and Epidemiology, London School of Hygiene and Tropical Medicine, Keppel Street, London WC1E 7HT.

\section{References}

Acheson, E. D. (1967). Medical Record Linkage. Oxford University Press: London.

Adelstein, A. M. (1976). Policies of the Office of Population Censuses and Surveys. British Journal of Preventive and Social Medicine, 30, 1-10.

Alderson, M. R. (1965). The Accuracy of Certification of Death, and the Classification of the Underlying Cause of Death from the Death Certificate. MD Thesis: London.

Alderson, M., and Whitehead, F. (1974). Reviews of United Kingdom Statistical Sources Volume II. Edited by W. F. Maunder. Heinemann Educational Books: London.
Allen-Price, E. D. (1960). Uneven distribution of cancer in west Devon with particular reference to the divers water supplies. Lancet, 1, 1235-1238.

Beral, V. (1974). Cancer of the cervix: a sexually transmitted infection? Lancet, 1, 1037-1040.

Beral, V. (1975). An epidemiological study of recent trends in ectopic pregnancy. British Journal of $\mathrm{Ob}$ stetrics and Gynaecology, 82, 775-782.

Beral, V. (1976). Cardiovascular disease mortality trends and oral contraceptive use in young women. Lancet, 2, 1047-1052.

Beral, V., Fraser, P., and Chilvers, C. (1978). Does pregnancy protect against ovarian cancer? Lancet, 1, 1083-1087.

Chilvers, C. (1978). Regional mortality 1969-73. Population Trends, 11, 16-20.

Chilvers, C., and Adelstein, A. (1978). Cancer mortality: the regional pattern. Population Trends, 12, 4-9.

Clifford, R. E., Smith, J. W. G., Tillett, H. E., and Wherry, P. J. (1977). Excess mortality associated with influenza in England and Wales. International Journal of Epidemiology, 6, 115-128.

Crawford, M. D., Gardner, M. J., and Morris, J. N. (1971). Cardiovascular disease and the mineral content of drinking water. British Medical Bulletin, 27, 21-24.

Doll, R. (1974). Surveillance and monitoring. International Journal of Epidemiology, 3, 305-314.

Donnan, S., and Haskey, J. (1977). Alcoholism and cirrhosis of the liver. Population Trends, 7, 18-24.

Elwood, P. C., St. Leger, A. S., and Morton, M. (1977). Mortality and the concentration of elements in tap water in county boroughs in England and Wales. British Journal of Preventive and Social Medicine, 31, 178-182.

Farr, W. (1868). Narrative of the proceedings at General Register Office during the Cholera epidemic 1866. Supplement to the 29th Report of the Registrar General, Appendix II. HMSO: London.

Fox, J. F. (1977). Occupational mortality 1970-72. Population Trends, 9, 8-15.

Galbraith, N. S., and Berrie, J. R. H. (1978). Statutory notification and surveillance of infectious diseases. Health Trends, 10, 32-34.

Guide to Official Statistics (1978). HMSO: London.

Haenszel, W. (1950). A standardised rate for mortality defined in units of lost years of life. American Journal of Public Health. 40, 17-26.

Health and Safety Executive (1976). Threshold limit values for 1976. Guidance note EH 15/76. HMSO: London.

Hill, G. B., and Adelstein, A. M. (1967). Cohort mortality from carcinoma of the cervix. Lancet, 2, 605-606.

Hill, M. J., Hawksworth, G., and Tattersall, G. (1973). Bacteria, nitrosamines and cancer. British Journal of Cancer, 24, 562-567.

Hobbs, M. S. T., Fairbairn, A. S., Acheson, E. D., and Baldwin, J. A. (1976). Study of disease associations from linked records. British Journal of Preventive and Social Medicine, 30, 141-150. 
Hoover, R., Mason, T. J., McKay, F. W., and Fraumeni, J. F., Jr (1975). Cancer by county: New resource for etiologic clues. Science, 189, 1005-1007.

Institute of Cancer Research (1976). Serial Mortality Tables. Institute of Cancer Research: London.

Kleinman, J. C. (1977). Age-adjusted mortality indexes for small areas: applications to health planning. American Journal of Public Health, 67, 834-840.

Langmuir, A. D. (1963). The surveillance of communicable diseases of national importance. New England Journal of Medicine, 268, 182-192.

Leck, I., and Record, R. G. (1966). Seasonal incidence of anencephalus. British Journal of Preventive and Social Medicine, 20, 67-75.

Lloyd, O. L. (1978). Respiratory cancer clustering associated with localised industrial air pollution. Lancet, 1, 318-320.

Macfarlane, A. (1977). Daily mortality and environment in English conurbations. 1. Air pollution, low temperature, and influenza in Greater London. British Journal of Preventive and Social Medicine, 31, 54-61.

Ministry of Health. (1954). Mortality and Morbidity during the London Fog of December 1952. Reports on Public Health and Medical Subjects, No. 95. HMSO: London.

Office of Population Censuses and Surveys (1973). Cohort Studies: New Developments. Studies on Medical and Population Subjects, No. 25. HMSO: London.

Office of Population Censuses and Surveys (1975). Cancer Mortality, England and Wales, 1911-1970. HMSO: London.

Office of Population Censuses and Surveys (1978a). Trends in Mortality, England and Wales, 1950-75. HMSO: London.

Office of Population Censuses and Surveys (1978b). Mortality Surveillance, England and Wales, 1968-1976. OPCS: London.
Office of Population Censuses and Surveys (1978c). Occupational Mortality, Decennial Supplement, England and Wales, 1970-1972. HMSO: London.

Reid, D. D. (1969). The beginnings of bronchitis. Proceedings of the Royal Society of Medicine, 62, 311-316.

Reid, D. D., and Grimley Evans, J. (1970). New drugs and changing mortality from non-infectious disease in England and Wales. British Medical Bulletin, 26, 191-196.

Reid, D. D. (1975). International studies in epidemiology. American Journal of Epidemiology, 102, 469-476.

Rose, G. (1966). Cold weather and ischaemic heart disease. British Journal of Preventive and Social Medicine, 20, 97-100.

Royal College of Physicians (1962). Smoking and Health. Pitman Medical: London.

Waterhouse, J. A. H. (1974). Cancer Handbook of Epidemiology and Prognosis. Churchill Livingstone: Edinburgh.

Weatherall, J. A. C., and Haskey, J. C. (1976). Surveillance of malformations. British Medical Bulletin, 32, 39-44.

Weatherall, J. A. C. (1978). Congenital malformations: surveillance and reporting. Population Trends, 11, 27-29.

World Health Organisation (1967). International Classification of Diseases, 8th revision. WHO: Geneva.

Wynne-Griffith, G. (1976). Cancer surveillance with particular reference to the uses of mortality data. International Journal of Epidemiology, 5, 69-76.

Yerushalmy, J. (1951). A mortality index for use in place of the age-adjusted death rate. American Journal of Public Health, 41, 907-922. 